\title{
Interaction between the atmospheric boundary layer and a stand- alone wind turbine in Gansu-Part I: Field measurement
}

\author{
DeShun $\mathrm{Li}^{1,2,3}$, Tao Guo ${ }^{1}$, YinRan $\mathrm{Li}^{1,2,3}$, JinSen $\mathrm{Hu}^{1}$, Zhi Zheng ${ }^{1,2,3}$, Ye $\mathrm{Li}^{4,5,6^{*}}$, YuJia $\mathrm{Di}^{4}$, \\ WenRui $\mathrm{Hu}^{{ }^{7 *}}$, and RenNian $\mathrm{Li}^{1,2,3^{*}}$ \\ ${ }^{1}$ School of Energy and Power Engineering, Lanzhou University of Technology, Lanzhou 730050, China; \\ ${ }^{2}$ Gansu Provincial Technology Centre for Wind Turbines, Lanzhou 730050, China; \\ ${ }^{3}$ Key Laboratory of Fluid Machinery and Systems, Lanzhou 730050, China; \\ ${ }^{4}$ School of Naval Architecture, Ocean and Civil Engineering, Shanghai Jiao Tong University, Shanghai 200240, China; \\ ${ }^{5}$ State Key Laboratory of Ocean Engineering, School of Naval Architecture, Ocean and Civil Engineering, Shanghai Jiao Tong University, \\ Shanghai 200240, China; \\ ${ }^{6}$ Collaborative Innovation Center for Advanced Ship and Deep-Sea Exploration, Shanghai Jiao Tong University, Shanghai 200240, China; \\ ${ }^{7}$ Institute of Mechanics, Chinese Academy of Sciences, Beijing 100080, China
}

Received January 30, 2018; accepted April 8, 2018; published online May 31, 2018

\begin{abstract}
Experiments and numerical simulations of the wake field behind a horizontal-axis wind turbine are carried out to investigate the interaction between the atmospheric boundary layer and a stand-alone wind turbine. The tested wind turbine $(33 \mathrm{~kW})$ has a rotor diameter of $14.8 \mathrm{~m}$ and hub height of $15.4 \mathrm{~m}$. An anti-icing digital Sonic wind meter, an atmospheric pressure sensor, and a temperature and humidity sensor are installed in the upstream wind measurement mast. Wake velocity is measured by three US CSAT3 ultrasonic anemometers. To reflect the characteristics of the whole flow field, numerical simulations are performed through large eddy simulation (LES) and with the actuator line model. The experimental results show that the axial velocity deficit rate ranges from $32.18 \%$ to $63.22 \%$ at the three measuring points. Meanwhile, the time-frequency characteristics of the axial velocities at the left and right measuring points are different. Moreover, the average axial and lateral velocity deficit of the right measuring point is greater than that of the left measuring point. The turbulent kinetic energy (TKE) at the middle and right measuring points exhibit a periodic variation, and the vortex sheet-pass frequency is mostly similar to the rotational frequency of the rotor. However, this feature is not obvious for the left measuring point. Meanwhile, the power spectra of the vertical velocity fluctuation show the slope of -1 , and those of lateral and axial velocity fluctuations show slopes of -1 and $-5 / 3$, respectively. However, the inertial subranges of axial velocity fluctuation at the left, middle, and right measuring points occur at 4, 7, and $7 \mathrm{~Hz}$, respectively. The above conclusion fully illustrates the asymmetry of the left and right measuring points. The experimental data and numerical simulation results collectively indicate that the wake is deflected to the right under the influence of lateral force. Therefore, wake asymmetry can be mainly attributed to the lateral force exerted by the wind turbine on the fluid.
\end{abstract}

wind power, atmospheric turbulence effects, velocity measurements, turbulent wakes, large-eddy simulations

PACS number(s): $89.30 . \mathrm{Ee}, 42.68 . \mathrm{Bz}, 47.80 . \mathrm{Cb}, 47.27 . w b, 47.27 . \mathrm{ep}$

Citation: D. S. Li, T. Guo, Y. R. Li, J. S. Hu, Z. Zheng, Y. Li, Y. J. Di, W. R. Hu, and R. N. Li, Interaction between the atmospheric boundary layer and a standalone wind turbine in Gansu—Part I: Field measurement, Sci. China-Phys. Mech. Astron. 61, 094711 (2018), https://doi.org/10.1007/s11433-018$9219-\mathrm{y}$

*Corresponding authors (Ye Li, email: ye.li@sjtu.edu.cn; WenRui Hu, email: wrhu@imech.ac.cn; RenNian Li, email: lirn@lut.edu.cn) 


\section{Introduction}

Wind turbines work in the complex atmospheric boundary layer where the variation in wind speed and direction can affect the characteristics of inflow turbulence. At the same time, wind-turbine blades are under a strong transient and alternating aerodynamic load exerted by vortex shedding. The wake characteristics of wind turbines must be studied to obtain the theoretical basis for the design of wind turbines with aerodynamic shapes, the calculation of wind-turbine performance, and the establishment of an accurate wake model.

Numerous studies on wind-turbine wake have been conducted within the past 20 years. The numerical study of the wind-turbine wake is mainly based on blade element momentum (BEM) theory and computational fluid dynamics (CFD) theory. For example, Vermeer et al. [1] studied the aerodynamics of horizontal axis wind-turbine wakes. They focused on the physics of power extraction by wind turbines and investigated near- and far-wake regions. Crespo et al. [2] provided an overview and analysis of different wake-modeling methods that may be used as prediction and design tools for wind turbines and wind farms. Snel [3] described and reviewed the engineering and scientific methods that are based on aeroelastic computer programs and fundamental Euler and Navier-Stokes (NS) equations. Zhao et al. [4] used the robust CLORNS code to predict the complex unsteady aerodynamic characteristics of a rotor flow field. Hansen et al. [5] reviewed the simple aerodynamic BEM and previous works that applied CFD to investigate the aerodynamics of wind-turbine rotors. Okulov et al. [6] focused on the socalled blade element theory and the Kutta-Joukowsky theorem, as well as on the development of Joukowsky's rotor vortex theory. Sanderse et al. [7] provided an overview of related research and summarized the CFD method from the aspects of governing equations, rotor modeling, boundary conditions, and wake modeling. Mo et al. [8,9] conducted large eddy simulation (LES) to study the effect of wind speed variation on the wake instability of the National Renewable Energy Laboratory (NREL) phase VI wind turbine. Ashton et al. [10] numerically studied the stability of the wind-turbine hub vortex. Several measurement techniques, such as hot-wire anemometry and particle image velocimetry (PIV), are widely used to measure the wake characteristics of wind turbines. Vermeer [11] used hot-wire anemometry to measure the near-wake of a model wind turbine and capture the trajectory of the tip vortex. Grant et al. [12-14] conducted a wind tunnel test to study the shape and velocity distribution of the tip vortex of a wind turbine with yaw. Haans et al. [15$18]$ investigated the tip vortex trajectory and wake of wind turbines. Medici et al. $[19,20]$ measured the shedding and movement of wake vortices to validate the BEM theory. To improve the BEM theory, Clausen et al. [21] used mean flow results to investigate the velocity of wind-turbine wake. They used a conventional three-hole yawmeter and an X-probe hot-wire anemometer to measure velocity. They then compared the experimental results from two different measuring techniques to analyze the BEM theory's ability to predict the performance of wind turbines. Ebert et al. [22-24] conducted numerous experimental studies on wind turbines by employing hot-wire anemometry and concluded a series on the formation and development of a model for the near-wake in horizontal-axis wind. Parkin et al. [25] used PIV to study the mean velocity distribution of the near-wake region and improve the accuracy of the prediction model. Their experimental results showed that a wake vortex with a high tip speed ratio expands and contracts. They then used test data obtained through PIV to calculate the geometry of windturbine wake at a tip Reynolds number of 2600 to 16000 . Whale [26] studied wake features in detail by comparing the results obtained by a sophisticated free-wake numerical code with wake measurements obtained through PIV. The wake trajectory inferred from PIV measurements was in good agreement with the theoretical results of the free-wake model. Hu et al. [27] conducted a wind tunnel test to study the dynamic wind load and wake characteristics of a wind turbine in the atmospheric boundary layer. The wake characteristics of an actuator disc and a model wind turbine were compared and analyzed by Aubrun et al. [28] and Lignarolo et al. [29] through wind tunnel experiments. Muller et al. [30] used hot-wire anemometry to measure the wake velocity of a wind turbine and applied spectral analysis to study the wake's nonstationarity. Zhang et al. [31] applied PIV to measure the near-wake and applied spectral analysis to study the energy distribution and turbulence characteristics of a wind turbine in an atmospheric boundary layer (ABL) wind tunnel.

National Renewable Energy Laboratory, Energy Research Centre of the Netherlands, Delft University of Technology, Imperial College, Rutherford Appleton Laboratory, and Risø National Laboratory have collaborated to conduct field experiments on wind turbines and to create a database $[32,33]$. Barthelmie et al. [34] used sonic detection and ranging (SODAR) to measure the velocity distribution of the windturbine wake and compared their measurements with the results of a relative numerical simulation. DAN-AERO MW experiments [35] were performed over the period of 2007 to 2009. Systematic field tests have been conducted on megawatt wind turbines. In these experiments, blade surface pressure, inflow, wake, and aero-acoustic parameters were recorded to investigate three-dimensional effects, dynamic stall characteristics, blade surface-boundary layer transition, wake characteristics, and aero-acoustics. However, the detailed experimental data remain undisclosed. The Los Alamos National Laboratory in the United States conducted field experiments on a wind turbine with a diameter of $4.5 \mathrm{~m}$. 
The wind turbine was installed with eight CSAT3 anemometers combined with two three-dimensional LF-PIV systems to enable the measurement of inflow and wake velocity. Li et al. [36] performed a field experiment on a vertical-axis tidal power system and found that flow turbulence characteristics exert a resonance effect on the impeller system. $\mathrm{Li}$ et al. [37] performed field experiments to investigate the effects of turbulence intensity and wind shear on the wake characteristics of wind turbines.

The majority of related studies have mainly performed wind tunnel tests and numerical simulations, whereas few have conducted field experiments. Given the complexity and instability of the atmospheric boundary layer flow and the influence of the scale effect, wind tunnel tests cannot reflect the real working conditions of wind turbines. Therefore, the flow characteristics of the actual wind field must be investigated through field experiments.

A field experimental platform was established by Lanzhou University of Technology, Lanzhou, China, to investigate wind-turbine aerodynamics. Experimental studies on blade surface pressure and wake velocity have been conducted on the platform [38-40]. In this study, we performed field measurements to analyze the time series, deficit rate, and turbulence characteristics of the wake velocity of wind turbines.

\section{Experimental equipment}

Figure 1 shows the field experimental system, which comprises a $33 \mathrm{~kW}$ wind turbine, a $30 \mathrm{~m}$ high anemometer tower, a wake-velocity measurement platform, and a controlling device.

\subsection{Experimental wind turbine}

The $33 \mathrm{~kW}$ wind turbine is a horizontal-axis two-bladed upwind-type turbine. It has variable pitch angles, a diameter of $14.8 \mathrm{~m}$, and National Advisory Committee for Aeronautics (NACA) series blades. It is located in Jing-tai
County, Gansu Province, China. In this region, the annual average wind velocity reaches 5.4 and $7 \mathrm{~m} / \mathrm{s}$ at the heights of 10 and $40 \mathrm{~m}$, respectively. Additionally, the effective wind velocity $(4-25 \mathrm{~m} / \mathrm{s})$ lasts for $7160 \mathrm{~h}$ annually. The average win energy density is $354 \mathrm{~W} / \mathrm{m}^{2}$, which meets the requirements for field tests.

\subsection{Measurement of wake velocity}

The wake-velocity measurement platform comprises an $18 \mathrm{~m}$ high hydraulic lift, the anemometer installation platform, and three sets of the US CSAT3 three-dimensional ultrasonic wind velocity instrument. The height of the hydraulic lifting platform can be adjusted remotely, and horizontal movement is realized by wheels installed under the base ground. Three CSAT3 anemometers are located in the vertical $(x-z)$ plane (parallel to the rotor-swept plane) one rotor diameter downstream. The \#1 (left) anemometer is also located in the vertical $(x-y)$ plane (the axial plane of the rotor, $z=0$ ). The \#2 (middle) and \#3 (right) anemometers are in the same horizontal line as the \#1 anemometer. The \#1, \#2, and \#3 anemometers are separated by a distance of $2.5 \mathrm{~m}$, and the vertical position of the anemometers is $2 \mathrm{~m}$ lower than that of the center of the rotor. Figure 2 shows the locations of the three measuring points. Three-dimensional ultrasonic anemometers are used to measure velocity components in three directions at a point in the flow field, and the digital signal is output through the RS-232 interface. In this study, $W, U$, and $V$ designate the vertical, rotor axis, and lateral directions, respectively. The measurement range for all the three velocity components is $\pm 65.535 \mathrm{~m} / \mathrm{s}$ with the resolution of $1 \mathrm{~mm} / \mathrm{s}$ in the $W$ and $V$ directions and $0.5 \mathrm{~mm} / \mathrm{s}$ in the $U$ direction. The measurement error is less than $4 \mathrm{~cm} / \mathrm{s}$ in the $W$ and $V$ directions and $2 \mathrm{~cm} / \mathrm{s}$ in the $U$ direction.

To ensure the accurate positions of the measuring points, the wake survey site is hardened with concrete. By taking the projection of the wind-turbine rotor centered on the ground as the origin and the north direction as the baseline, we defined the sector ranging from $35^{\circ}$ eastward to $20^{\circ}$ westward as the wake measurement region. Lines are drawn every $5^{\circ}$.

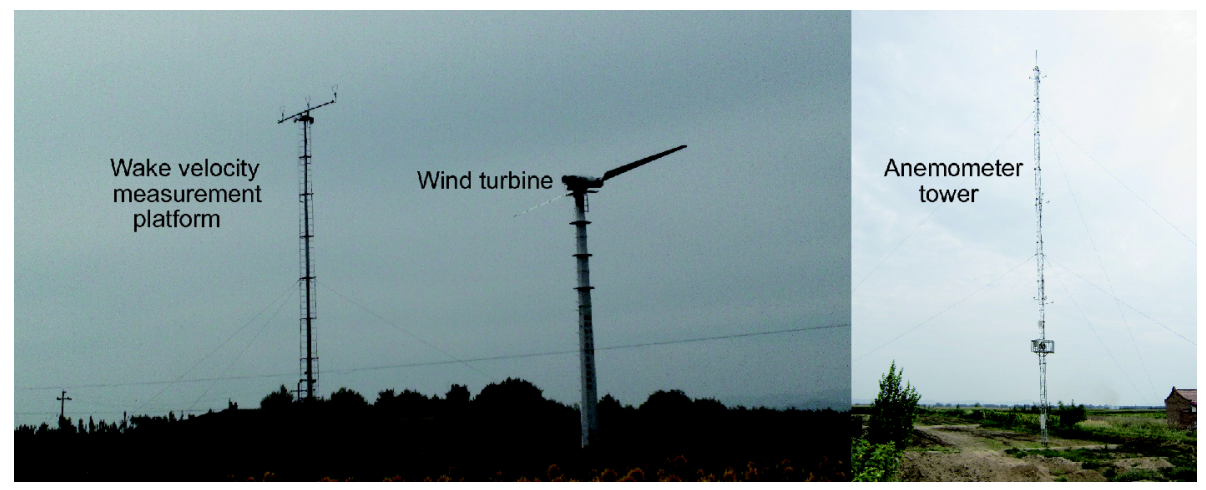

Figure 1 (Color online) Field experiment system, wake-velocity measurement platform, wind turbine, and anemometer tower. 


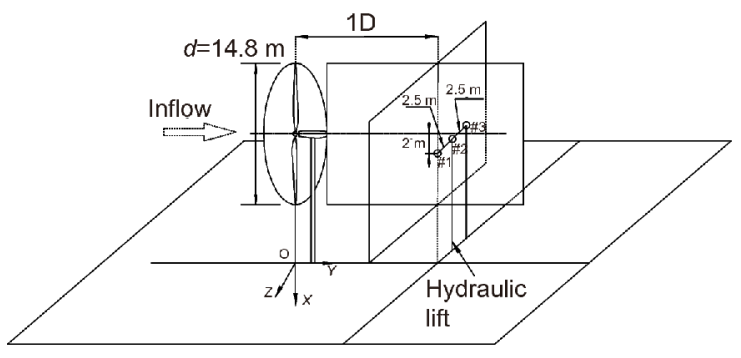

(a)

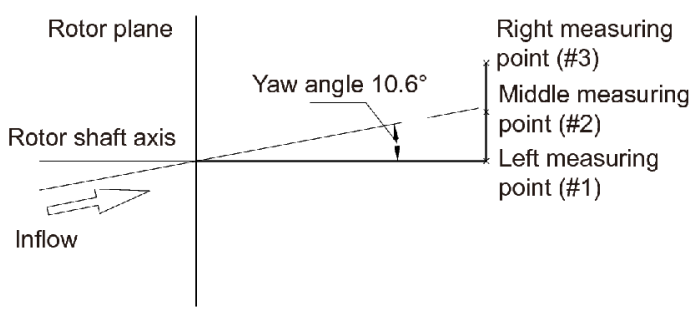

(b)

Figure 2 Schematic of measurement locations (a), top view of measurement locations (b).

Meanwhile, the distances of $0.6 D, 1 D, 1.5 D$, and $2 D$, where $D$ refers to wind-turbine diameter, are marked on each angle line to determine the position of the lifting platform. Finally, we can determine the spatial location of the anemometers by combining the horizontal position and the lifting height of the lifting platform. The location of the anemometers are shown in Figure 2.

When performing wake measurements, we first move the lifting platform to a calibrated section normal to the wind direction. After the platform is adjusted to a horizontal position, it is raised to the measuring height $(13.4 \mathrm{~m})$ where the test is conducted.

\subsection{Measurement of inflow parameters}

On the $30 \mathrm{~m}$ anemometer tower, an anti-icing digital Sonic wind meter is installed at the height of the rotor center to measure wind velocity and direction. An atmospheric pressure sensor and a temperature and humidity sensor are also installed on the anemometer tower to measure local atmospheric pressure, air temperature, and humidity.

\subsection{Measurement of other wind-turbine parameters}

The active yaw system is based on the worm gear mechanism, which is driven by a direct current motor. Taking account of the transmission ratio, the yaw angle is measured by counting the number of turns of the motor shaft. The blade setting angle is controlled by the electrohydraulic switching valve and is measured by a displacement sensor. Rotor velocity is measured by an electrooptical sensor stuck onto the hub. Blade surface pressure is measured by a pressure sensor.

\section{Wake propagation}

\subsection{Experimental conditions}

Field experiments on wake velocity behind the wind turbine are carried out. The sampling time for inflow parameters, the operating condition parameters of the wind turbine, and wake velocity is $6 \mathrm{~s}$. The sampling frequency of inflow parameters and operating condition parameters of the wind turbine is $5 / 3 \mathrm{~Hz}$, and the sampling frequency of the wake velocity is $20 \mathrm{~Hz}$. The following is the analysis of a set of experimental results.

Table 1 shows the conditions of an experiment. In this table, $V_{\mathrm{IN}}$ is inflow velocity, $\Psi$ is the angle between inflow and the north direction, $P_{0}$ is atmospheric pressure, $T_{0}$ is atmospheric temperature, $\varphi$ is atmospheric humidity, $n$ is the rotor rotational velocity, $\gamma$ is the angle between the windturbine axis and the south direction, and $\beta$ is the adjustment value of the pitch angle. The time series of the inflow velocity $V_{\mathrm{IN}}$ and the wake-velocity components ( $W$ for vertical direction, $U$ for rotor axis direction, and $V$ for lateral direction) are shown in Figure 3.

Table 1 shows that the inflow parameters and the operating

Table 1 Experimental conditions

\begin{tabular}{|c|c|c|c|c|c|c|c|c|c|c|c|c|}
\hline \multirow{2}{*}{\multicolumn{2}{|c|}{ Test condition }} & \multicolumn{10}{|c|}{ Test value } & \multirow{2}{*}{ AVG } \\
\hline & & 1 & 2 & 3 & 4 & 5 & 6 & 7 & 8 & 9 & 10 & \\
\hline \multirow{5}{*}{ Inflow } & $V_{\mathrm{IN}}(\mathrm{m} / \mathrm{s})$ & 4 & 4 & 4 & 4 & 3.9 & 4 & 4 & 4.1 & 4.1 & 4.1 & 4.02 \\
\hline & $\Psi\left(^{\circ}\right)$ & 351 & 351 & 351 & 348 & 348 & 351 & 351 & 351 & 351 & 351 & 350.4 \\
\hline & $P_{0}(\mathrm{~Pa})$ & 82820 & 82820 & 82820 & 82820 & 82820 & 82820 & 82820 & 82820 & 82820 & 82820 & 82820 \\
\hline & $T_{0}\left({ }^{\circ} \mathrm{C}\right)$ & 23.1 & 23.1 & 23.1 & 23.1 & 23.1 & 23.1 & 23.1 & 23.1 & 23.1 & 23.1 & 23.1 \\
\hline & $\varphi(\%)$ & 52.6 & 52.5 & 52.5 & 52.5 & 52.5 & 52.5 & 52.5 & 52.4 & 52.4 & 52.4 & 52.48 \\
\hline \multirow{3}{*}{ Turbine } & $n(\mathrm{r} / \mathrm{min})$ & 54.5 & 54.5 & 54.45 & 54.45 & 54.45 & 54.45 & 54.45 & 54.45 & 54.45 & 54.5 & 54.47 \\
\hline & $\Gamma\left(^{\circ}\right)$ & 181 & 181 & 181 & 181 & 181 & 181 & 181 & 181 & 181 & 181 & 181 \\
\hline & $B\left({ }^{\circ}\right)$ & 48 & 48 & 48 & 48 & 48 & 48 & 48 & 48 & 48 & 48 & 48 \\
\hline
\end{tabular}




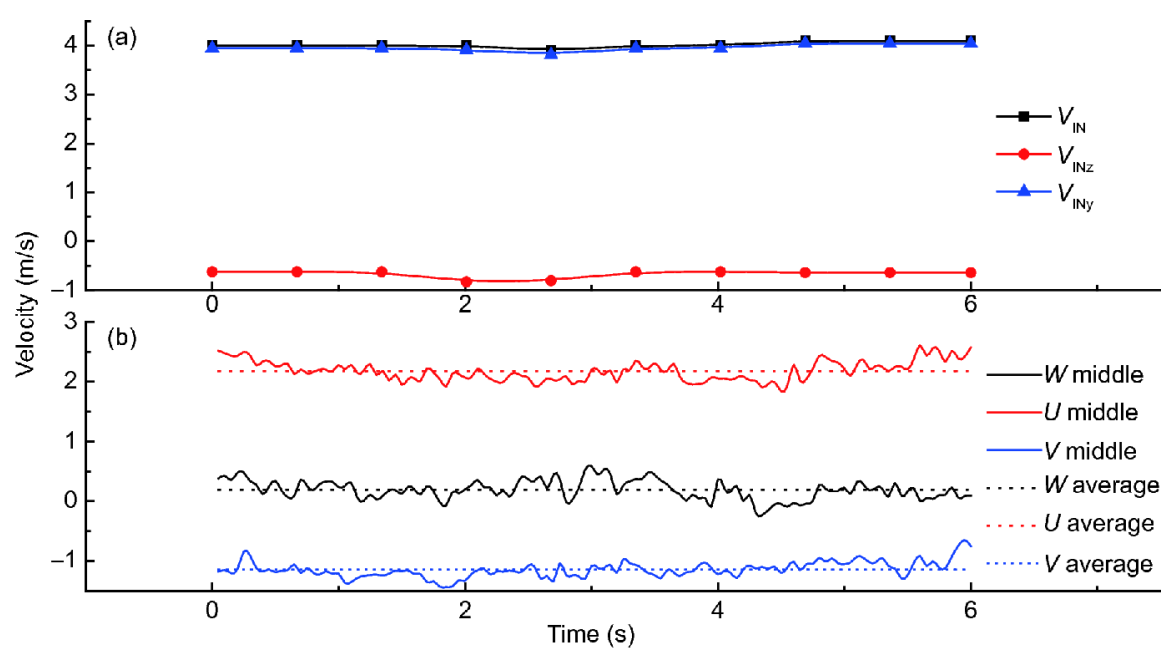

Figure 3 (Color online) Inflow velocity and its lateral and axial (rotor axis) components (a). Axial, lateral, and vertical velocity components at the middle anemometer and their average value (b).

parameters of the wind turbine are stable under the experimental conditions. Specifically, the mean wind velocity is $4.02 \mathrm{~m} / \mathrm{s}$ with the upper deviation of $0.08 \mathrm{~m} / \mathrm{s}(+1.99 \%)$ and the lower deviation of $0.12 \mathrm{~m} / \mathrm{s}(-2.99 \%)$. Furthermore, the average wind direction is $350.4^{\circ}$ with the upper deviation of $0.6^{\circ}(+0.17 \%)$ and the lower deviation of $2.4^{\circ}(-0.68 \%)$. Other inflow parameters are almost constant within the sampling time. Given the angle $\gamma=181^{\circ}$, we conclude that the yaw angle is $10.6^{\circ}$. Considering the positional arrangement of the three anemometers under this operating condition, anemometer \#2 is located roughly at the intersection of the vertical plane of inflow through hub center and the vertical plane (parallel to the rotor-swept plane) one rotor diameter downstream, as shown in Figure 2(b).

\subsection{Axial velocity deficit rate in the wake}

To analyze the variation in wake velocity, the axial velocity deficit rate $D_{y}$ is defined as:

$D_{y}=\frac{\left(\bar{V}_{\mathrm{IN} y}-U\right)}{\overline{\mathrm{IN}}_{\mathrm{IN}}}$

where $V_{\text {INy }}$ represents the axial velocity of the inflow and $U$ represents the axial velocity of the wake.

The measurement results show that the average axial wake velocity $U$ is $2.18 \mathrm{~m} / \mathrm{s}$ with a deficit of $44.81 \%$. The average lateral wake velocity $V$ at the middle measuring point is -1.14 , which shows an increase of $60.13 \%$. The average vertical wake velocity $W$ is $0.13 \mathrm{~m} / \mathrm{s}$. Compared with the lateral inflow velocity, the lateral wake velocity has increased. This behavior reflects the wake's expansion characteristics. The deficit rate of axial velocity deficit at the left, middle, and right points fluctuates in the range of $32.18 \%$ $55.79 \%, 39.97 \%-53.37 \%$, and $34.99 \%-63.22 \%$, respectively.

\subsection{Characteristics of turbulent flow in the wake}

The variation in turbulent velocity in the wake will affect the load of wind-turbine blades. In particular, the sheddingtrailing vortex will exert alternating load on the blade. Alternating load, in turn, will shorten fatigue life.

Here, we analyze the characteristics of the turbulent flow in the wake from the point of turbulent kinetic energy (TKE) [36], which is given as:

$\operatorname{TKE}(t)=\frac{1}{2}\left[u^{\prime}(t)^{2}+v^{\prime}(t)^{2}+w^{\prime}(t)^{2}\right]$,

where $u^{\prime}(t), v^{\prime}(t)$, and $w^{\prime}(t)$ are the fluctuation values of axial velocity, lateral velocity, and vertical velocity, respectively.

Coherent turbulence kinetic energy (CTKE) is obtained with eq. (3), which reflects the cross-axis properties that enable the investigation of the temporally and spatially coherent turbulence structure:

$$
\begin{aligned}
\operatorname{CTKE}(t)= & \frac{1}{2}\left[\left(u^{\prime}(t) v^{\prime}(t)\right)^{2}\right. \\
& \left.+\left(u^{\prime}(t) w^{\prime}(t)\right)^{2}+\left(v^{\prime}(t) w^{\prime}(t)\right)^{2}\right]^{1 / 2} .
\end{aligned}
$$

The horizontal flow angle $\psi^{\prime}(t)$ and the vertical flow angle $\alpha^{\prime}(t)$ of the wake-velocity fluctuation can be calculated with eqs. (4) and (5). Thus, the direction-variant characteristics of turbulence fluctuation at a specific point in the wake can be obtained:

$\psi^{\prime}(t)=\tan ^{-1}\left(v^{\prime}(t) / u^{\prime}(t)\right)$,

$\alpha^{\prime}(t)=\tan ^{-1}\left(w^{\prime}(t) / u^{\prime}(t)\right)$.

Figure 4 shows the time-domain characteristics of turbulence velocity fluctuation and the $u^{\prime}, v^{\prime}, w^{\prime}$, TKE, CTKE, $\psi^{\prime}(t)$, and $\alpha^{\prime}(t)$ of wake-velocity fluctuation as measured by the middle anemometer. TKE presents a certain periodic regularity. Six peaks appeared within the sampling time of $6 \mathrm{~s}$, as shown in the TKE and CTKE curves. This result 

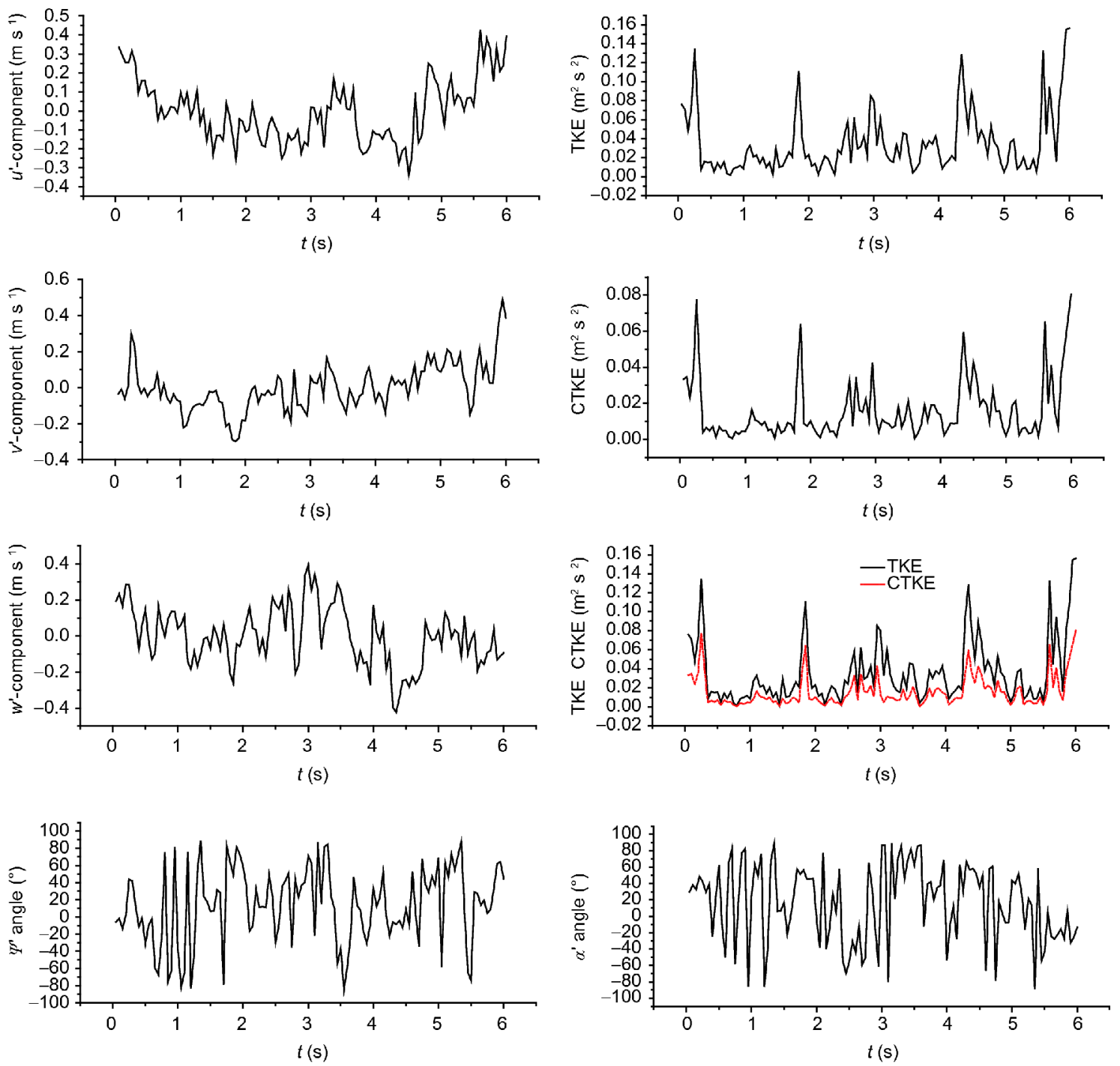

Figure 4 (Color online) Full records of the observed time series of $u^{\prime}, v^{\prime}, w^{\prime}$, TKE, CTKE, $\psi^{\prime}$, and $\alpha^{\prime}$ for the middle measuring point.

illustrates that the passage frequency of the trailing vortex is close to the rotational frequency of the rotor. The waveform of the TKE curve is similar to that of the CTKE curve. Moreover, the $\psi^{\prime}(t)$ and $\alpha^{\prime}(t)$ of the wake-velocity fluctuation change in the range of $-90^{\circ}$ to $90^{\circ}$.

Figure 5 shows the results of fast Fourier transform of wake-velocity fluctuation $\left(u^{\prime}, v^{\prime}\right.$ and $\left.w^{\prime}\right)$ at the middle measuring point. The characteristics of the turbulence power spectra at the measuring point are described in this figure. The power spectra of velocity fluctuation in the $V$ - and $U$ directions present slopes of -1 and $-5 / 3$, respectively. The power spectra of velocity fluctuation in the $W$-direction has a slope of -1 . The results show that the classical production and inertial subranges for turbulence are regions that follow power-law scaling with slopes of -1 and $-5 / 3$, respectively. The results reveal that, in the wake, the characteristics of the production and inertial subranges will be reflected by the turbulence components in the lateral and axial directions.
The figure indicates that the inertial subrange moves backward and does not appear in the vertical direction. This behavior shows that the energy transfer and dissipation of turbulence in the vertical direction occur at high frequencies. In other words, in the region of the wake within one dimension, turbulent flow mainly relies on large-scale vortex movement. TKE transports energy through inertia, and its dissipation is negligible.

Figure 6 shows the autocorrelation curves of the $\psi^{\prime}(t)$ and $\alpha^{\prime}(t)$ of the wake-velocity fluctuation at the middle measuring point. Their autocorrelation is poor. The poor autocorrelation of the two parameters indicate that velocity fluctuation has a random direction. Nevertheless, it is consistent with the rule of flow-angle autocorrelation under natural wind conditions.

Figure 7 shows the probability distributions of $\psi^{\prime}(t)$ and $\alpha^{\prime}(t)$ for the middle measuring point. The red line represents Gaussian distribution. The probability density distribution in 

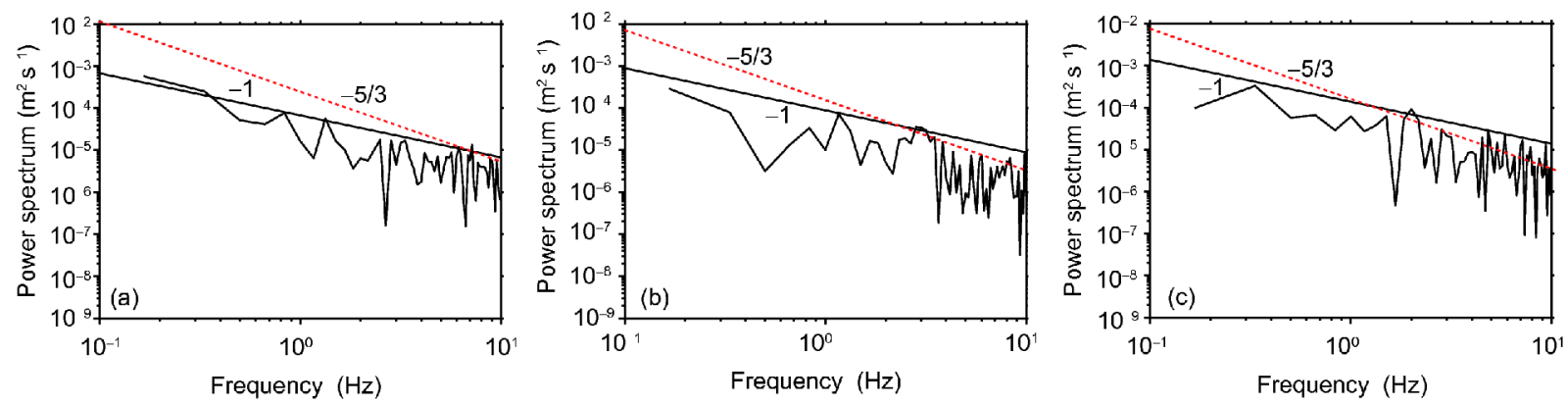

Figure 5 (Color online) Power spectrum of velocity fluctuations $v^{\prime}$ (a), $u^{\prime}$ (b), and $w^{\prime}$ (c) for the middle measuring point.
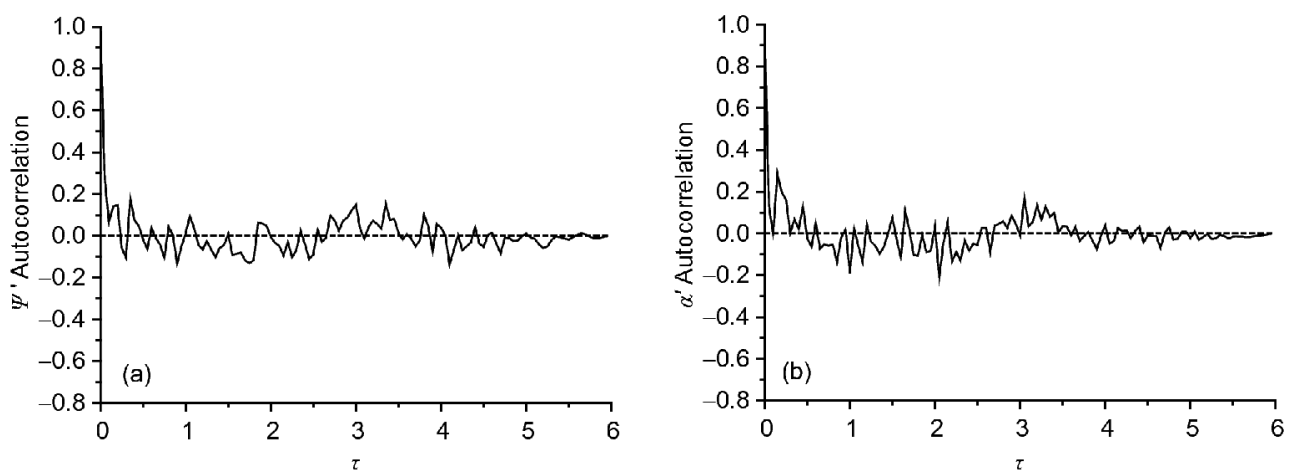

Figure 6 Autocorrelation curves of $\psi^{\prime}$ (a) and $\alpha^{\prime}$ (b) for the middle measuring point.
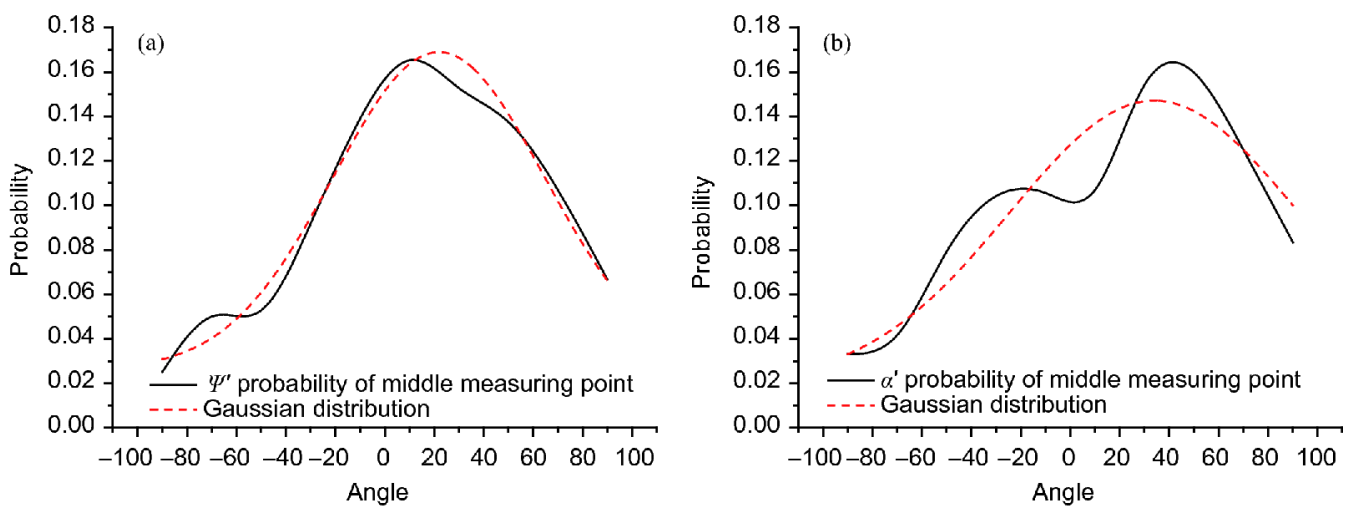

Figure 7 (Color online) Probability distributions of $\psi^{\prime}$ (a) and $\alpha^{\prime}$ (b) for the middle measuring point.

Figure 7(a) is unimodal and is in accordance with Gaussian distribution. Furthermore, the maximum probability distribution mainly lies in the range of $0^{\circ}$ to $20^{\circ}$. By contrast, the probability distribution in the right figure is bimodal, and the maximum probability distribution mainly lies in the range of $20^{\circ}$ to $60^{\circ}$.

\subsection{Analysis of wake asymmetry}

To analyze wake asymmetry, the relative value $R$ of left measuring point and right measuring point are respectively defined as:

$$
\begin{aligned}
& R_{\text {left }, X}=\frac{X_{\text {left }}-X_{\text {middle }}}{X_{\text {middle }}}, \\
& R_{\text {right }, X}=\frac{X_{\text {right }}-X_{\text {middle }}}{X_{\text {middle }}},
\end{aligned}
$$

where $X$ is given by the deficit rates of axial velocity and lateral velocity; the values of TKE, CTKE; and the probability distributions of $\psi^{\prime}(t)$ and $\alpha^{\prime}(t)$.

Figure 8 shows the time-domain and frequency domain features of the relative axial velocity of the right and left measuring points in each rotational period over five continuous rotational periods. The relative axial velocity of the 


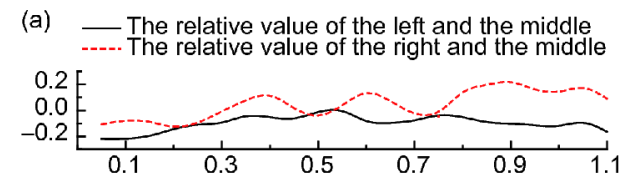

(b) - The relative value of the left and the middle

0.12 F
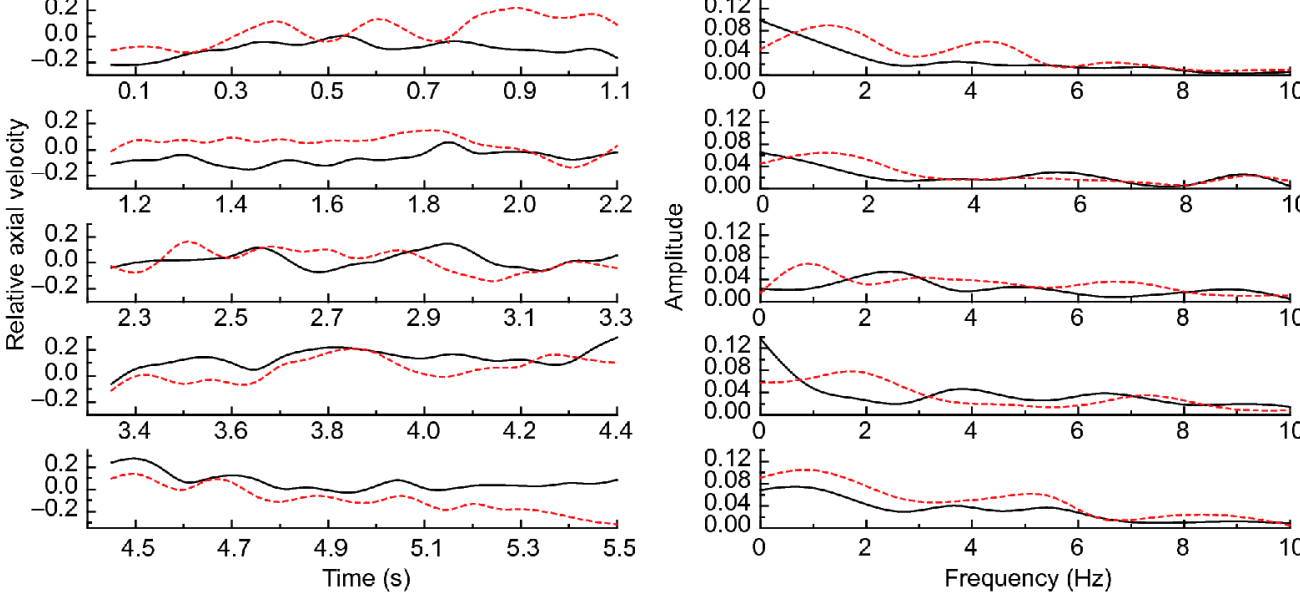
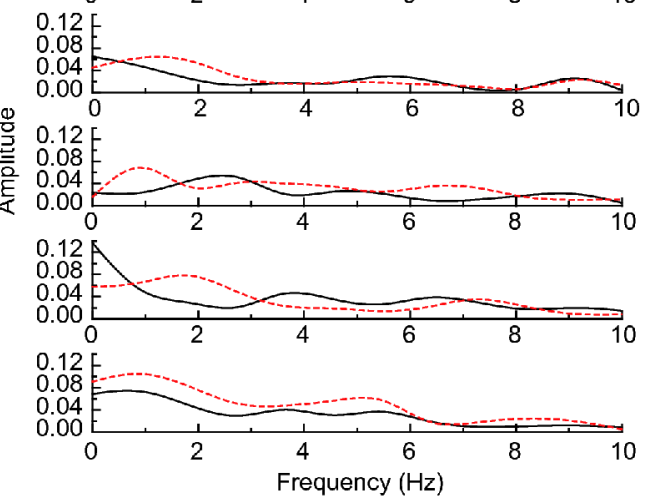

Figure 8 (Color online) Time domain (a) and frequency domain (b) of relative axial velocity.

left and right measuring points exhibit the same trend in the time-domain graph. However, the fluctuation of the right point is more intense than that of the left. Meanwhile, the relative axial velocity of the right measuring point is larger than that of the left measuring point during the first two rotational periods but exhibit opposite results during the fourth and fifth rotational periods. At the same time, the overall velocity fluctuation of the right measuring point is larger than that of the left measuring point. The frequency domain graph shows that the difference between the left and right measuring points mainly appears in the low-frequency band. Moreover, the first, second, and fourth circles in Figure 8(b) indicate that the amplitude of the right measuring point exceeds $1.8 \mathrm{~Hz}$. The peak of the third and fifth circles occur at $0.9 \mathrm{~Hz}$, whereas that of the left measuring point is absent.

\section{Numerical simulation}

The flow field cannot be completely described by limited numbers of experimental points. To reflect the characteristics of the whole flow field and to supplement our experimental results, we performed numerical simulations. In this research, we use the LES method and actuator line model to simulate the experimental unit. The calculation process is divided into two parts. First, the neutral atmosphere boundary layer is generated on the basis of the LES. Then, the rotor, which is replaced with the actuator line model, is placed in the atmospheric boundary layer to simulate interaction between the atmospheric boundary layer and a standalong wind turbine. The detailed calculation results and analysis have been presented in another article [41].

Turbulence is divided into large-scale and small-scale vortices through filtering. Large-scale vortices are directly solved by N-S equations. Small-scale vortices are modeled by the subgrid scale (SGS) model. To simulate the neutral atmosphere boundary layer, we need to consider the effects of the buoyancy effect, Coriolis force effect, and surface heat flux. Therefore, the filtered continuity equation is

$\frac{\partial \tilde{u}_{i}}{\partial x_{j}}=0$

The filtered momentum transport equation is

$$
\begin{aligned}
\frac{\partial \tilde{u}_{i}}{\partial t}+\frac{\partial}{\partial x_{j}}\left(\tilde{u}_{j} \tilde{u}_{i}\right)= & -2 \varepsilon_{i j k} \Omega_{j} \tilde{u}_{k}-\frac{\partial \widetilde{p}}{\partial x_{i}} \\
& -\frac{1}{\rho_{0}} \frac{\partial}{\partial x_{i}} \widetilde{p}_{0}(x, y)-\frac{\partial \widetilde{\tau}_{i j}}{\partial x_{j}} \\
& -g\left(\frac{\widetilde{\theta}-\theta_{0}}{\theta_{0}}\right) \delta_{i j}+\frac{f_{\varepsilon}}{\rho_{0}},
\end{aligned}
$$

where $\Omega_{j}$ is the rotation rate vector, which is defined as $\Omega=$ $\omega[0, \cos (\varphi), \sin (\varphi)]$, and $\omega$ is the planetary rotation rate. $\phi$ is the latitude. The gravitational constant $g$ is $9.81 \mathrm{~m} / \mathrm{s}^{2} . \tilde{\theta}$ is the resolved potential temperature, and $\theta_{0}=300 \mathrm{~K}$ is the reference temperature. $f_{\varepsilon}$ is the body force field exerted by the actuator line turbine model. The Smagorinsky model is used to close SGS stress. The model is

$\widetilde{\tau}_{i j}=-2\left(c_{\mathrm{s}} 4\right)^{2}\left(2 s_{i j} s_{i j}\right)^{\frac{1}{2}}=-2 v^{\mathrm{SGS}} s_{i j}$,

where SGS viscosity, $v^{\mathrm{SGS}}$, is obtained in standard Smagorinsky [42] mode. $C_{\mathrm{s}}$ is the Smagorinsky constant. $\Delta$ represents filter scales.

$S_{i j}=\frac{1}{2}\left(\frac{\partial \tilde{u}_{i}}{\partial x_{j}}+\frac{\partial \tilde{u}_{j}}{\partial x_{i}}\right)$.

The filtered potential temperature transport equation is

$\frac{\partial \tilde{\theta}}{\partial t}+\frac{\partial}{\partial x_{j}}\left(u_{j} \tilde{\theta}\right)=-\frac{\partial q_{j}}{\partial x_{j}}$,

where $q_{j}$ represents the temperature transport by molecular 
and SGS turbulence effects.

$q_{j}=-k^{\mathrm{SGS}} \frac{\partial \tilde{\theta}}{\partial x_{j}}$,

$k^{\mathrm{SGS}}=\frac{v^{\mathrm{SGS}}}{P r_{\mathrm{t}}}$,

where the turbulent Prandtl number $P r_{\mathrm{t}}$ is $1 / 3$.

The length, width, and height of the calculation field are 3 , 3 , and $1 \mathrm{~km}$, respectively. The wind turbine is located in the center of the computational domain $(x=1500, y=1500, z=0)$. The mesh is locally encrypted near the wind turbine. The inflow velocity and yaw angle are $4.02 \mathrm{~m} / \mathrm{s}$ and $10.6^{\circ}$, respectively, and are the same as those used for the experimental setups. The dark area in Figure 9 is the mesh encryption area. In this article, the mesh near the wind turbine is encrypted six times with the minimum mesh size of $0.52 \mathrm{~m}$.

Table 2 shows the comparison of time-average axial wind velocity at the left, middle, and right measuring points behind the rotor at $700 \mathrm{~s}$ of the simulation and at $6 \mathrm{~s}$ of the experiment. This table shows that the error of inflow velocity between the experiment and CFD method is $4.71 \%$. The errors of the left, middle, and right measuring points are $1.239 \%, 29.180 \%$, and $31.846 \%$, respectively, and are mainly caused by ignoring the hub effect in the numerical simulation. Ignoring the hub effect decreases the wake-velocity deficit, especially for the middle and right measuring

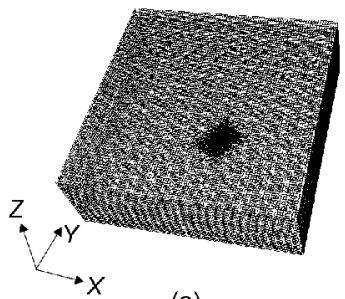

(a)

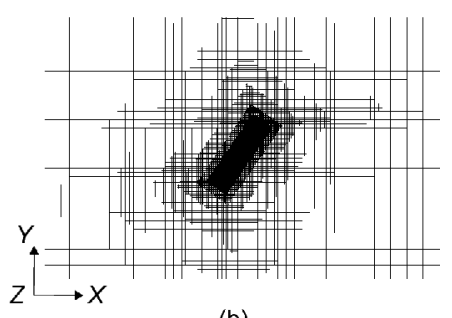

(b)
Figure 9 Computing mesh (a) and mesh horizontal section (b).

points. As shown in Figure 10, the right measuring point is close to the central vortex, and the left measuring point is distant from the central vortex. The right measuring point is more easily affected by the center of the vortex than the left measuring point. Therefore, the error of the right measuring point is higher than that of the left measuring point.

Figure 10 shows the distribution of instantaneous horizontal velocity at the height of the central rotor. When the wind flows through the rotor, the wind turbine absorbs some of the inflow energy. As a result, the inflow energy becomes deficient and velocity decreases. Wake deflection occurs because of wind-turbine yaw. In addition, the left and right velocity distribution of the centerline at the wake is asymmetric. The deficit of the right-side wake is higher than that of the left-side wake, and the right-side wake expands more than the left-side wake.

Table 2 Comparison between numerical results and experimental data

\begin{tabular}{|c|c|c|c|c|}
\hline Index & $u$ of inflow velocity & $u$ of left measuring point & $u$ of middle measuring point & $u$ of right measuring point \\
\hline Experiment $(\mathrm{m} / \mathrm{s})$ & 3.950 & 2.180 & 2.183 & 2.151 \\
\hline Numerical result $(\mathrm{m} / \mathrm{s})$ & 4.136 & 2.207 & 2.820 & 2.836 \\
\hline Error $(\%)$ & 4.709 & 1.239 & 29.180 & 31.846 \\
\hline
\end{tabular}

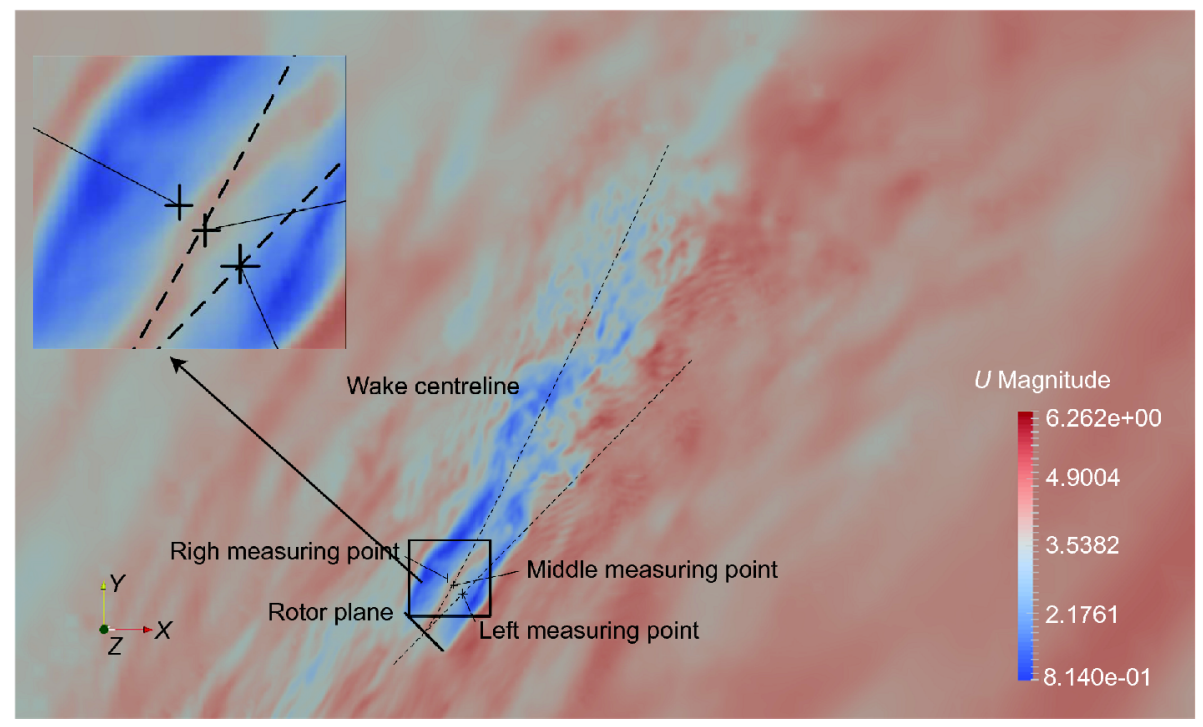

Figure 10 Velocity nephogram of horizontal section. 
To further analyze asymmetry in the structure of the windturbine wake, the time-averaged axial velocity horizontal profile at each measuring-point height is extracted at multiple locations upstream and downstream of the rotor $(-1 \leq$ $L / D \leq 5)$. In Figure 11, $D$ is the diameter of the rotor, and the ordinate $L / D$ represents the relative distance from the center of the rotor. The abscissa $U / U_{0}$ represents the dimensionless axial velocity, and $U_{0}$ is the average axial velocity of the inflow $\left(U_{0}=4.136 \mathrm{~m} / \mathrm{s}\right)$. The figure shows that the wake center is consistently skewed to the right as distance increases. At the distances of $1 D, 2 D$, and $3 D$ to the rotor plane, the wake velocity is asymmetric along the wake center, and the right deficit is greater than the left deficit. This difference is caused by the lateral force exerted after the wind-turbine yaw. The effect of velocities gradually weakens with increasing rotor plane distance. The velocity deficit on the center line of the wake is small, and the wake-velocity curve of the wind turbine shows an M-shaped distribution when hub impact is ignored. When the distance from the rotor plan increases, the M-shaped distribution gradually turns into a unimodal distribution. The three measuring points are marked at $1 D$ distances, and the dashed line at each point represents the fluctuation range of the axial velocity $U / U_{0}$. The fluctuation range is approximately 0.299 $0.615,0.426-0.877$, and 0.492-0.790.

The small deficit of the middle measuring point is mainly attributed to the ignorance of the hub effect, and that of the right measuring point is mainly attributed to the influence of lateral force. The right measuring point is closer to the central vortex than the left point. This position indicates that the right point is more sensitive to the central vortex and reveals the reasons for the higher velocity deficit of the right measuring point than that of the left measuring point.

\section{Discussion}

This article investigates wind-turbine wake by analyzing the velocity deficits and turbulence characteristics of experi-

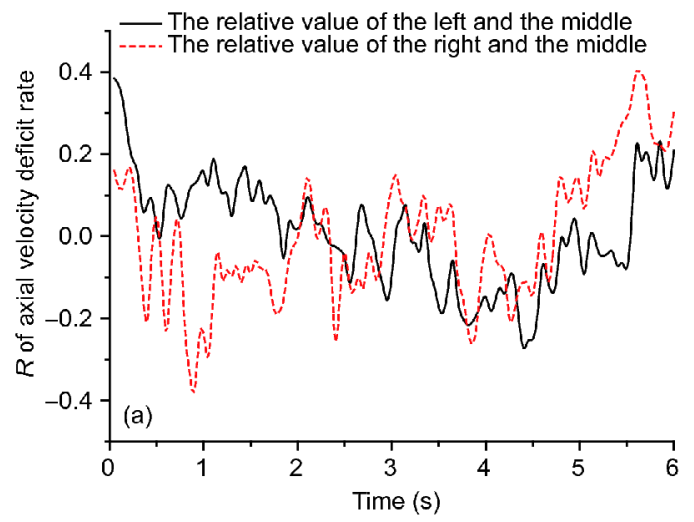

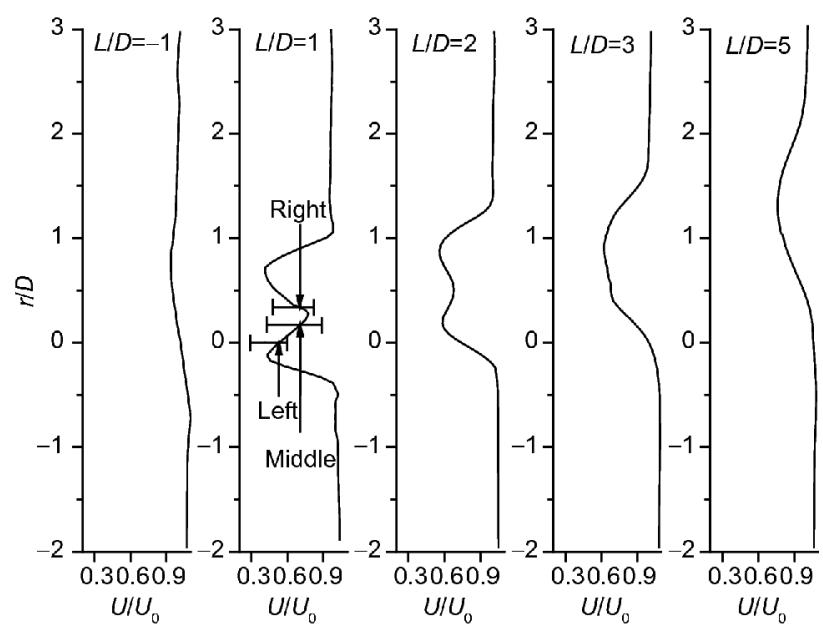

Figure 11 Spanwise distribution of time-averaged axial velocity.

mental measuring points. Moreover, the experimental results for the wake-velocity field are supplemented by numerical simulation results. Geometrically, the middle measuring point of the wakes should be approximately located at the central location of the three measuring points. Thus, the wake features of the right and left measuring points should be similar. However, the axial velocities at the left and right measuring points exhibit different time-frequency characteristics and obvious asymmetry. The asymmetry of the wake is discussed on the basis of the experimentally obtained velocity and turbulence characteristics of the left and right measuring points.

To reflect wake asymmetry, Figure 12 shows the relative value of the axial velocity deficit rate and lateral velocity deficit rate. Figure 12(a) shows that the relative value considerably differs within $0-2.5$ and 5-5.7 s. However, the relative values of the deficit rate from 2.5 to $5 \mathrm{~s}$ are negligibly different. The experimental results show that the average deficit rate of relative axial velocity at the right measuring point $(0.03)$ is higher than that at the left measuring point (0.01). This difference is attributed to the asymmetry between the left and right measuring points.

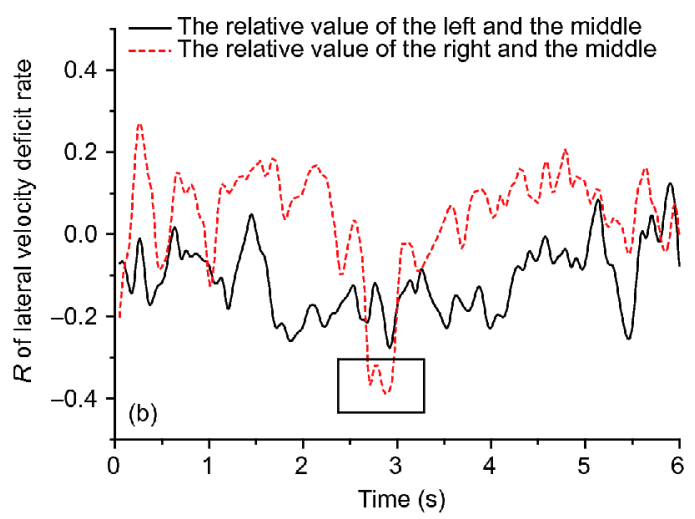

Figure 12 (Color online) Relative values of axial velocity deficit rate (a) and lateral velocity deficit rate (b). 
Figure 12(b) shows the comparisons of lateral velocity deficit rate between the left and right measuring points. In this figure, the red line is consistently higher than the black line except at the point indicated by a box. This pattern indicates that the lateral deficit rate of the right measuring point is significantly higher than that of the left measuring point and further reflects the significant asymmetry of the wake in the lateral direction. The effect of vertical shear on wake asymmetry is negligible given the small rotor size of the wind turbine. Wake asymmetry is mainly caused by a lateral component that stems from the thrust that the rotor exerts on the fluid after wind-turbine yaw [43]. The lateral force causes the central vortex to deflect to the right and to then affect the right measuring point, thus resulting in the greater lateral velocity deficit of the right measuring point than that of the left. The sudden drop indicated by the box may be caused by strong turbulence at the right measuring point at that moment.

The comparisons between the relative CTKE (a) and TKE (b) values of the left and right measuring points are shown in Figure 13. This figure clearly reflects the differences between the left and right measuring points. These differences reflect the asymmetry of the wake. By comparing the two figures shown in Figure 13, we find that the peaks appear at the same moment and at the same measuring point. On the whole, we can see that the red line is higher than the black line except at individual moments, indicating that the TKE of the right measuring point is higher than that of the left measuring point. From a partial perspective, however, the relative value exhibits a drastic fluctuation during the periods of 0.5-2.5 and 5-5.7 s. Combining the figure with Figure 12(a) reveals that the regions with drastic fluctuations coincide with the regions where TKE drastically fluctuates. Thus, the sharp change in TKE influences the sharp change in axial velocity deficit rate and causes wake asymmetry.

The boxed region in Figure 13 shows that the TKE value of the right measuring point at the corresponding time is higher than that of the left measuring point. Moreover, it is main-
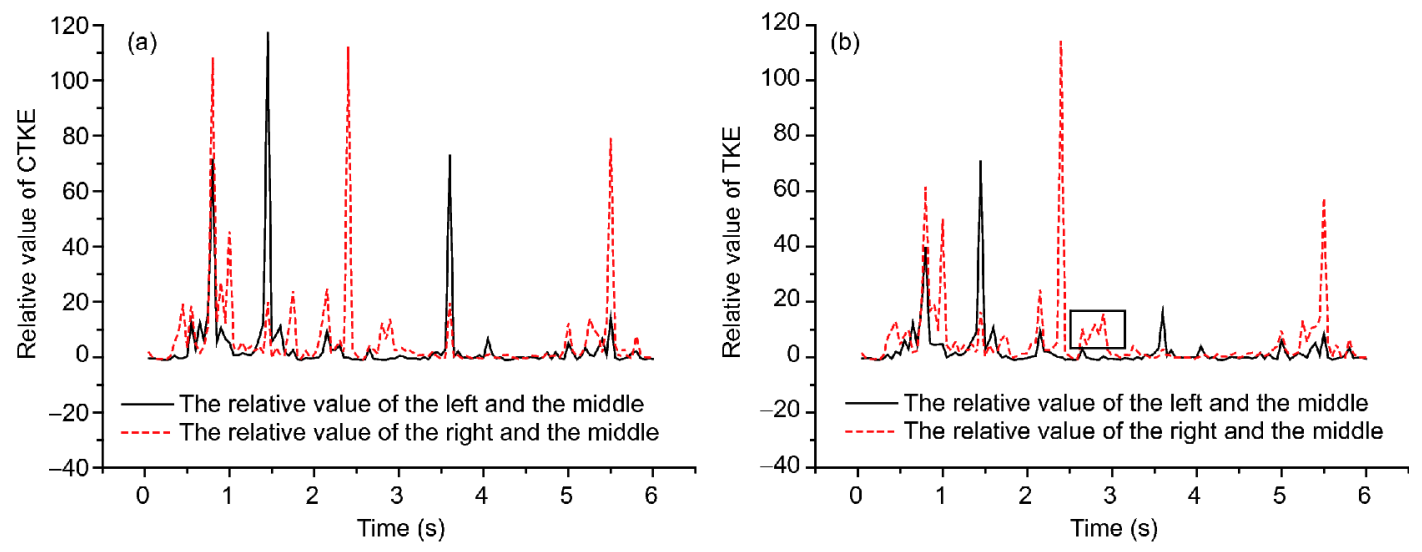

Figure 13 (Color online) Relative values of CTKE (a) and TKE (b). tained for a longer duration, indicating that large-scale turbulence exists at the right measuring point exists at the moment. The large-scale turbulence may cause a sudden drop in the lateral velocity deficit at the right measuring point in Figure 12.

Figure 14 shows the power spectra of the velocity fluctuation in the axial, lateral, and vertical directions of the left and right measuring points. Compared with that in the power spectrum of $u^{\prime}$ at the middle measuring point, the $-5 / 3$ region of the left measuring point is larger, indicating that the inertial subrange has moved forward. However, the inertial subrange of the right measuring point occurs almost at the same position as that of the middle measuring point, specifically, at $7 \mathrm{~Hz}$. The production subrange of turbulence at the right measuring point in the axial direction is larger than that of the left measuring point. Compared with the power spectrum of $v^{\prime}$ at the middle measuring point, the position of the inertial subrange of the three measuring points almost all occur at $4 \mathrm{~Hz}$. Furthermore, all of $w^{\prime}$ at the three measuring points have a slope of -1 , and the inertial subrange is absent. This result suggests that energy transfer and turbulence dissipation in the vertical direction occur at high frequencies. The inertial subrange of the vertical velocity spectrum of the atmospheric surface layer is mainly concentrated in the highfrequency region. Moreover, its shape remains basically unchanged, and its inertial subrange approximately appears at the position of $0.5 \mathrm{~Hz}$ [44]. In this research, the inertial subrange of the vertical velocity spectrum significantly moves backward after $10 \mathrm{~Hz}$. The backward movement of the subrange may be attributed to strengthened turbulence in the vertical direction after rotor rotation. In conclusion, the above analysis reflects the asymmetry of turbulence characteristics in the wake.

Figure 15 shows the relative value of the probability distributions of $\psi^{\prime}$ and $\alpha^{\prime}$. The probability distribution of the flow angle of the left measuring point is steadier than that at the right. The probability distribution of the flow angle of the right measuring point is high between $-90^{\circ}$ and $-50^{\circ}$, 

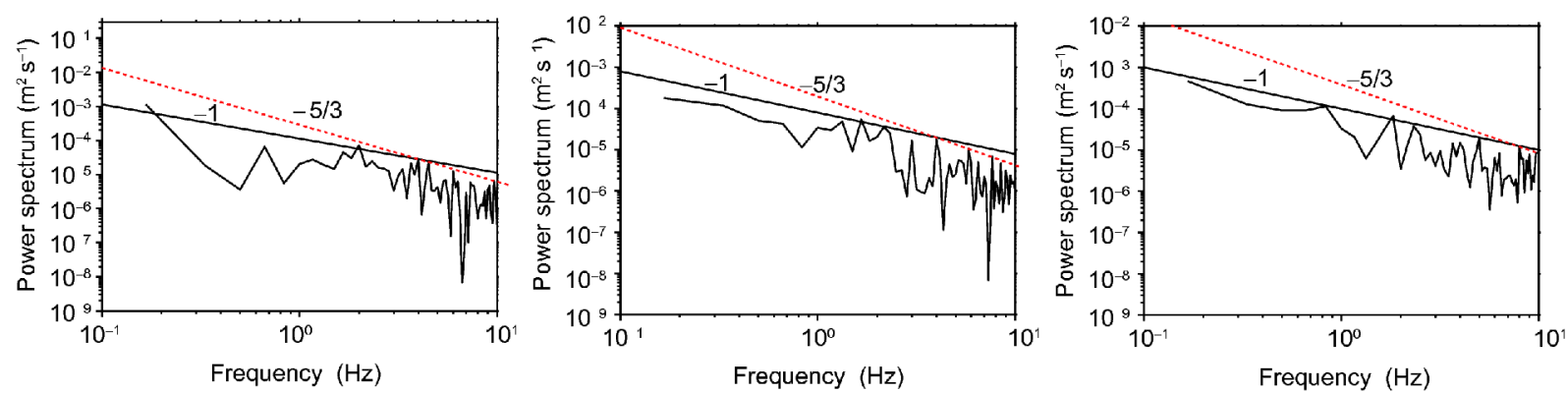

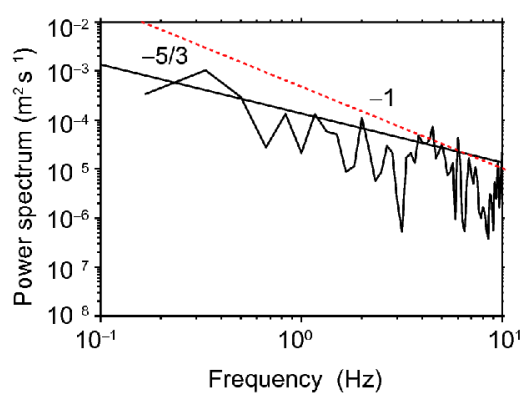

(a)

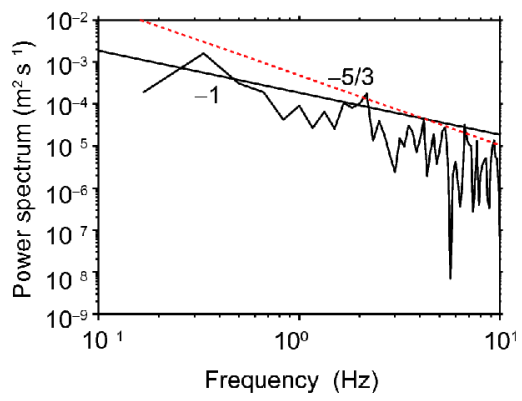

(b)

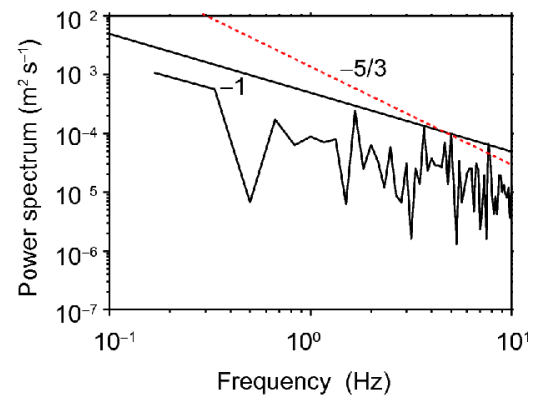

(c)

Figure 14 (Color online) Power spectrum of the velocity fluctuations $\left(v^{\prime}(\mathrm{a}), u^{\prime}(\mathrm{b}), w^{\prime}(\mathrm{c})\right)$ for left measuring point (up) and the right measuring point (down).
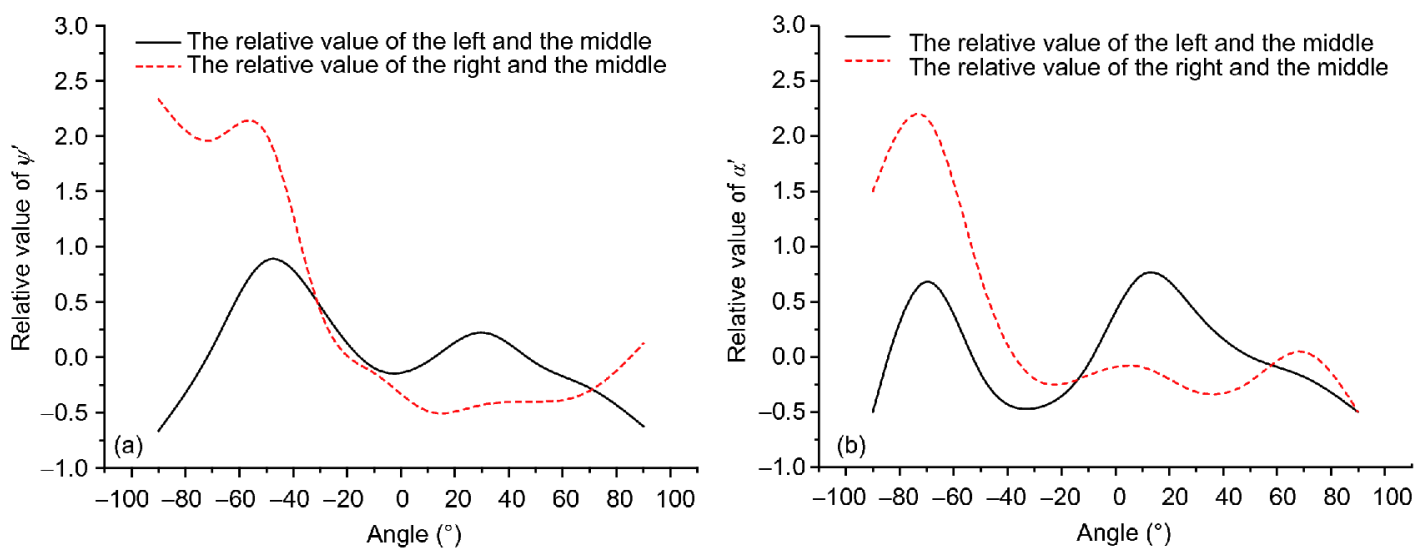

Figure 15 (Color online) Relative value of the probability distributions of $\psi^{\prime}$ (a) and $\alpha^{\prime}$ (b).

showing that flow at the right measuring point is more turbulent than that at the left. Therefore, the flow characteristics of the left and right points are asymmetrical.

Figure 16 shows the cross-correlation curves of $u^{\prime}$ and $\psi^{\prime}$ between the left and middle measuring points and between the right and middle measuring points. The figure shows that the cross-correlation coefficients of $u^{\prime}$ between the left and middle measuring points are high at $\tau=-4.2,-1.2,1.2$, and $4.2 \mathrm{~s}$ and show two sinusoidal periods. However, the crosscorrelation coefficients between the middle and right points are high at $\tau=-4.8,-2.8,-3.4,-0.4,1.2,3.6$, and $5.2 \mathrm{~s}$ and show three peaks and four troughs. The cross-correlation coefficients between the left and the middle of $\psi^{\prime}$ show four peaks. However, the cross-correlation coefficients between the middle and right measuring points show eight peaks. The analysis shows that the cross-correlation coefficient between the right and middle measuring points presents increased periodicity, and the cross-correlation coefficients of the two figures are similar. Considering that the right and middle measuring points may be in the same vortex system, then the right measuring point is closer to the center of the vortex than the left measuring points, as verified by numerical analysis. The results fully reflect the asymmetry of the velocity fluctuation and horizontal flow angle in the wake.

Figure 17 shows the cross-correlation curves of TKE between the left and middle measuring points and between the right and middle measuring points. In Figure 17(a), the correlation is weak and irregular, whereas the correlation shown in Figure 17(b) is strong with the periodicity of approximately $1.1 \mathrm{~s}$. This period corresponds with the rota- 

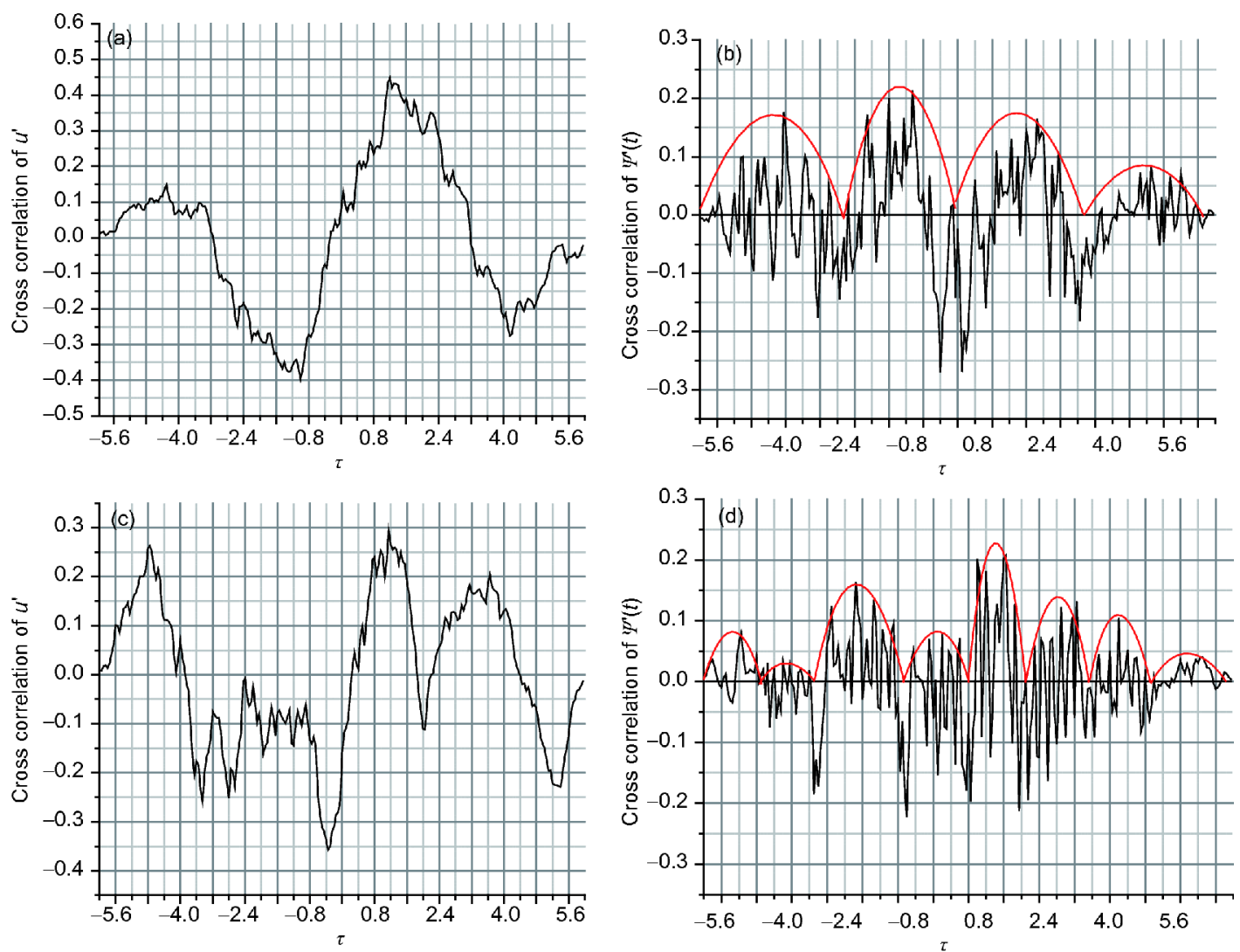

Figure 16 (Color online) Cross-correlation curves of $u^{\prime}$ and $\psi^{\prime}$ between the left and middle measuring points (a), (b) and between the right and middle measuring points (c), (d).
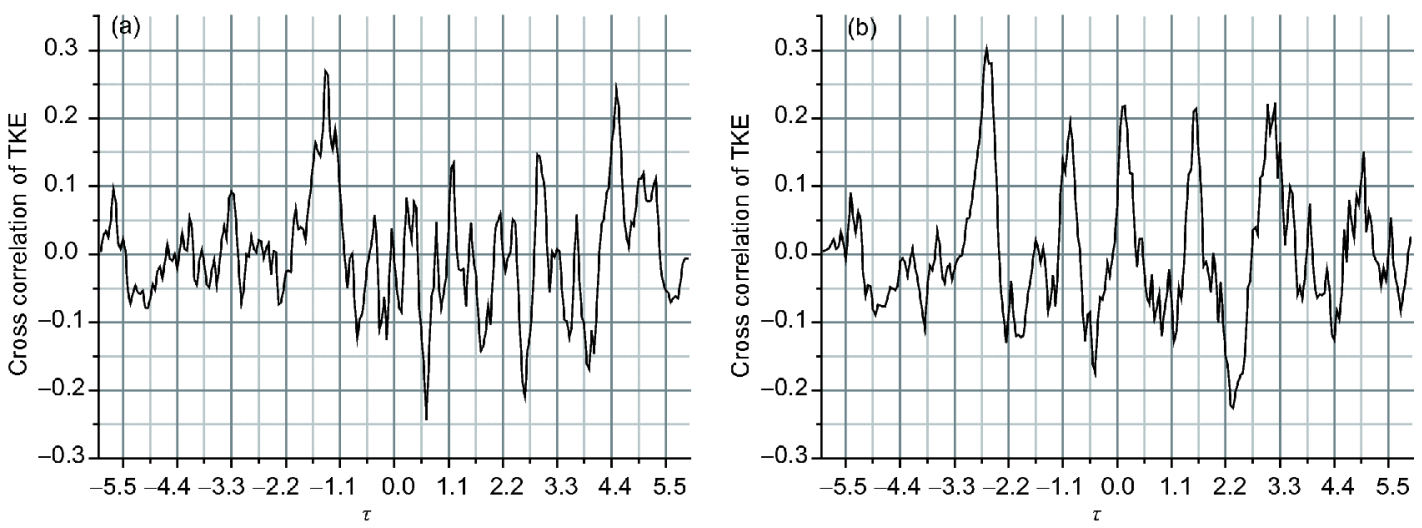

Figure 17 (Color online) Cross-correlation curve of TKE between the left and middle measuring points (a) and between the right and middle measuring points (b).

tional period of the rotor. The results of numerical simulation shown in Figure 10 collectively illustrate that the wake is deflected to the right under the action of lateral force.

\section{Conclusions}

This article conducts a new experiment and numerical simulation to further understand the asymmetric wake char- acteristics of a horizontal-axis wind turbine. Several conclusions can be drawn from the experimental data and numerical results.

(1) The axial velocity deficit rate is between $32.18 \%$ and $63.22 \%$ for the three measuring points. The average relative axial and lateral velocity deficit rates of the right measuring point are higher than those of the left measuring point. This result is verified by numerical simulations, which show that the velocity deficit and expansion of the right-side wake are 
larger than those of the left-side wake. Meanwhile, the right and middle measuring points are closer to the central vortex than the left measuring point. This result indicates that wake asymmetry is mainly caused by the lateral component, which stems from the thrust that the rotor exerts on the fluid after wind-turbine yaw.

(2) The probability distribution of the horizontal flow angle is consistent with Gaussian distribution, whereas that of the vertical flow angle presents a bimodal distribution at the middle measuring point. However, between $-90^{\circ}$ and $-50^{\circ}$, the probability distribution of the flow angle of the right measuring point is higher than that of the left measuring point. This result shows that flow at the right measuring point is more turbulent than that at the left measuring point.

(3) The power spectra and TKE of velocity fluctuation at the middle and right measuring points follow similar trends. The power spectra of vertical velocity fluctuation have a slope of -1 , and those of lateral and axial velocity fluctuations have slopes of -1 and $-5 / 3$. However, the inertial subrange of axial velocity fluctuation at the left, middle, and right measuring positions occur at different positions, i.e., 4, 7, and $7 \mathrm{~Hz}$, respectively. The TKE of the right measuring point is higher than that of the left measuring point. Nevertheless, the correlation between the right measuring point and the middle measuring point is stronger than that between other measuring points. Collectively, the experimental results and numerical simulations show that the middle and right measuring points are mainly affected by the central vortex. Furthermore, the wake is deflected to the right under the action of lateral force, thus resulting in wake asymmetry.

This work was supported by the National Basic Research Program of China (Grant No. 2014CB046201), the National Natural Science Foundation of China (Grant Nos. 51766009, 51566011, 51465033, and 51479114), the Thousand Talents Program (Grant No. NSFC-RCUK_EPSRC), the Platform Construction of Ocean Energy Comprehensive Supporting Service (2014) (Grant No. GHME2014ZC01), the High-tech Ship Research Projects Sponsored by MIITC Floating Support Platform Project (Grant No. 201622), and State Key Laboratory of Ocean Engineering at Shanghai Jiao Tong University.

1 L. J. Vermeer, J. N. Sørensen, and A. Crespo, Prog. Aerospace Sci. 39, 467 (2003).

2 A. Crespo, J. Hernández, and S. Frandsen, Wind Energy 18, 1 (2015).

3 H. Snel, Wind Energy 1, 46 (1998).

4 Q. Zhao, G. Zhao, B. Wang, Q. Wang, Y. Shi, and G. Xu, Chin. J. Aeronaut. 31, 214 (2018).

5 M. O. L. Hansen, J. N. Sørensen, S. Voutsinas, N. Sørensen, and H. A. Madsen, Prog. Aerospace Sci. 42, 285 (2006).

6 V. L. Okulov, J. N. Sørensen, and D. H. Wood, Prog. Aerospace Sci. 73, 19 (2015)

7 B. Sanderse, S. P. Pijl, and B. Koren, Wind Energy 14, 799 (2011).

8 J. O. Mo, A. Choudhry, M. Arjomandi, R. Kelso, and Y. H. Lee, J. Wind Eng. Ind. Aerodyn. 117, 38 (2013).

9 J. O. Mo, A. Choudhry, M. Arjomandi, and Y. H. Lee, J. Wind Eng. Ind. Aerodyn. 112, 11 (2013).

10 R. Ashton, F. Viola, F. Gallaire, and G. V. Iungo, J. Phys.-Conf. Ser.
625, 012033 (2015).

11 N. J. Vermeer, How Fast is a Tip Vortex? Technical Report (Delft University of Technology, 1995).

12 I. Grant, P. Parkin, and X. Wang, Exp. Fluids 23, 513 (1997).

13 I. Grant, M. Mo, X. Pan, P. Parkin, J. Powell, H. Reinecke, K. Shuang, F. Coton, and D. Lee, J. Wind Eng. Ind. Aerodyn. 85, 177 (2000).

14 I. Grant, and P. Parkin, Exp. Fluid. 28, 368 (2000).

15 W. Haans, T. Sant, G. van Kuik, and G. van Bussel, J. Sol. Energy Eng. 127, 456 (2005).

16 W. Haans, T. Sant, G. V. Kuik, and G. V. Bussel, in 31st European Rotorcraft Forum (Confederation of European Aerospace Societies, Tokyo, 2005), pp. 61.1-61.14.

17 W. Haans, T. Sant, G. van Kuik, and G. van Bussel, J. Sol. Energy Eng. 128, 472 (2006).

18 W. Haans, T. Sant, G. van Kuik, and G. van Bussel, Wind Energy 11, 245 (2008)

19 D. Medici, and P. H. Alfredsson, Wind Energy 9, 219 (2006).

20 D. Medici, and P. H. Alfredsson, Wind Energy 11, 211 (2008).

21 P. D. Clausen, D. M. Piddington, and D. H. Wood, J. Wind Eng. Ind. Aerodyn. 25, 189 (1987).

22 P. R. Ebert, and D. H. Wood, Renew. Energy 12, 225 (1997).

23 P. R. Ebert, and D. H. Wood, Renew. Energy 18, 513 (1999).

24 P. R. Ebert, and D. H. Wood, Renew. Energy 22, 461 (2001).

25 P. Parkin, R. Holm, D. Medici, in the Fourth International Symposium on Particle Image Velocimetry (DLR-Mitteilung, Gottingen, 2001), pp. $155-162$.

26 J. Whale, Investigating Fundamental Properties of Wind Turbine Wake Structure Using Particle Image Velocimetry, Technical Report (University of Edinburgh, 1996).

27 H. Hu, Z. Yang, and P. Sarkar, Exp. Fluid. 52, 1277 (2012).

28 S. Aubrun, S. Loyer, P. E. Hancock, and P. Hayden, J. Wind Eng. Ind. Aerodyn. 120, 1 (2013).

29 L. E. M. Lignarolo, D. Ragni, C. J. Ferreira, and G. J. W. van Bussel, J. Renew. Sustain. Energy 8, 023301 (2016).

30 Y. A. Muller, S. Aubrun, and C. Masson, Exp. Fluid. 56, 53 (2015).

31 W. Zhang, C. D. Markfort, and F. Porté-Agel, Exp. Fluid. 52, 1219 (2012).

32 J. G. Schepers, A. J. Brand, A. Bruining, J. M. R. Graham, M. M. Hand, D. G. Infield, H. A. Madsen, R. J. H. Paynter, and D. A. Simms, "Final report of IEA Annex XIV: Field Rotor Aerodynamics", No. ECN-C-97-027, 1997.

33 J. G. Schepers, A. Brand, and A. Bruining, "Final report of IEA Annex XVIII: Enhanced Field Rotor Aerodynamics DatabaseE", No. CN-C02-016, 2002.

34 R. J. Barthelmie, L. Folkerts, G. C. Larsen, K. Rados, S. C. Pryor, S. T. Frandsen, B. Lange, and G. Schepers, J. Atmos. Ocean. Technol. 23, 888 (2006).

35 A. M. Helge, B. Christian, S. P. Uwe, G. Mac, F. Peter, R. Jonas, E. Peder, L. Jesper, and J. Leo, the DAN-AERO MW Experiments Final Report (Danmarks Tekniske Universitet, 2010).

36 Y. Li, J. H. Yi, H. Song, Q. Wang, Z. Yang, N. D. Kelley, and K. S. Lee, Appl. Phys. Lett. 105, 023902 (2014).

37 Q. Li, T. Maeda, Y. Kamada, and N. Mori, Energy 134, 482 (2017).

38 R. N. Li, S. K. Yuan, L. J. Wei, D. S. Li, and Y. R. Li, J. Exp. Fluid. Mech. 26, 52 (2012).

39 D. S. Li, R. N. Li, X. Y. Wang, L. J. Wei, Y. R. Li, Y. Qiang, and Z. Q. Liu, Appl. Mech. Mater. 34, 1073 (2013).

40 D. S. Li, Y. R. Li, R. N. Li, S. J. Liu, Y. Li, and W. R. Hu, Sci. China Phys. Mech. Astron. 46, 124706 (2016).

41 Z. Zheng, Z. T. Gao, D. S. Li, R. N. Li, Y. Li, Q. H. Hu, and W. R. Hu, Sci. China Phys. Mech. Astron. http://doi.org/10.1007/s11433-0189214-1 (2018).

42 J. Smagorinsky, Mon. Wea. Rev. 91, 99 (1963).

43 P. M. O. Gebraad, F. W. Teeuwisse, J. W. van Wingerden, P. A. Fleming, S. D. Ruben, J. R. Marden, and L. Y. Pao, in the American Control Conference (ACC) (IEEE, Portland, 2014), pp. 3128-3134.

44 J. Wang, Sci. Atmosp. Sin. 16, 11 (1992). 